\title{
Exploring the Impacts of the Beginning of the COVID-19 Pandemic on Critical Care Physicians and the Delivery of Patient Care in Seven Countries: A Qualitative Interview-Based Study
}

\section{Jeanna Parsons Leigh ( $\nabla$ j.parsonsleigh@dal.ca )}

Dalhousie University https://orcid.org/0000-0002-8408-674X

\section{Chloe DeGrood}

Dalhousie University

\section{Alexandra Dodds}

Dalhousie University

Francesca Rubulotta

McGill University

Emily A. FitzGerald

Dalhousie University

\section{Sara J. Mizen}

Dalhousie University

Karla D. Krewulak

University of Calgary

Stephana J. Moss

Dalhousie University

Henry T. Stelfox

University of Calgary

Kirsten M. Fiest

University of Calgary

Research

Keywords: critical care, COVID-19, pandemic, interviews, preparedness

Posted Date: January 10th, 2022

DOI: https://doi.org/10.21203/rs.3.rs-1189373/v1

License: (9) This work is licensed under a Creative Commons Attribution 4.0 International License.

Read Full License 
Page $2 / 19$ 


\section{Abstract \\ Purpose}

To understand critical care physician experiences across multiple countries with the COVID-19 pandemic to inform future pandemic preparedness planning.

\section{Methods}

In this qualitative study, 16 critical care physicians (from seven countries) identified in convenience, purposive sampling took part in individual semi-structured interviews from April 7, 2020 to August 27, 2020 that captured the first wave of the pandemic. Open coding was conducted by two researchers who facilitated inductive thematic analysis.

\section{Results}

Key themes identified following thematic analysis included: 1) sourcing and implementation of trusted information; 2) health systems-level preparedness with accessible supports; 3 ) institutional adaptations including changes to patient care; 4) professional safety and occupational wellbeing; 5) triage and restricted visitation policies; and 6) managing personal familial responsibilities.

\section{Conclusion}

Perspectives of critical care physicians are important for ongoing pandemic planning and should be included in future pandemic policy development.

\section{Introduction}

Shortages in life-saving interventions including personal protective equipment, hospital and Intensive Care Unit (ICU) beds, and mechanical ventilators emerged globally in the first wave of the COVID-19 pandemic, driving many healthcare systems to rapidly develop triage plans to support the allocation of these limited resources [1-4]. Human resources have also been in high demand throughout the pandemic, resulting in healthcare professionals being redeployed to emergency departments and ICUs from other areas of the healthcare system to avoid acute services being overwhelmed by surges in COVID-19 case numbers $[5,6]$. A shift in emerging research has shown to have caused increased stress and strain for patients, families and healthcare professionals alike $[7,8]$.

Critical Care Medicine continues to be at the forefront of the pandemic response given that the most severely ill patients diagnosed with COVID-19 were and continue to be cared for in ICUs by critical care physicians [9]. Multiple studies conducted in Europe, North America and Asia have demonstrated the 
mental health burden of the pandemic on frontline healthcare professionals, especially those working in ICUs, where burnout was prevalent pre-COVID-19 [10-13]. In Canada, the fear of anticipated or realized resource strain during the pandemic was shown to heighten psychological distress in critical care physicians that included concerns related to personal and familial safety [2]. An international survey of critical care healthcare professionals also described how the COVID-19 pandemic has impacted resources, staffing, and patient care globally, demonstrating that interventions tailored to healthcare professionals' needs are required to ameliorate healthcare professionals stress and burnout from working during a pandemic [14]. Guidelines for preparation and management of ICUs during emergency situations such as the COVID-19 pandemic are increasingly being developed, and include specifics such as promoting infection prevention, increased infrastructure and staffing preparations, ICU capacity building, triage policies, and research development [15].

Research is emerging on the multifaceted impacts of the pandemic on critical care physicians, and the delivery of patient care [16]. Healthcare professionals are on the frontlines of public health crises and their perspectives are invaluable when assessing the impacts and areas of improvement required within healthcare systems. Therefore, the objective of this study was to understand how critical care physicians, their clinical practice, and the hospitals in which they work, were impacted by the initial stage of the COVID-19 pandemic across multiple countries to understand shared preparedness needs to inform future stakeholder driven pandemic preparedness planning for the COVID-19 pandemic and future pandemics.

\section{Methods}

\section{Study Design}

Two researchers $(\mathrm{CD}, \mathrm{CH})$ conducted a qualitative semi-structured interview study and reported it according to the Consolidated Criteria for Reporting Qualitative Research (COREQ) [Appendix 1]. The University of Calgary Conjoint Health Research Ethics Board (Ethics ID\#: REB20-0377) and Dalhousie University Research Ethics Board (Ethics ID\#: 2020-5106) approved this study.

\section{Participants}

FR applied a purposive and convenience sampling strategy using personal contacts to recruit critical care physicians from the United States of America (USA), Canada, Turkey, England, Scotland, Italy, Spain, and Pakistan. These countries were chosen based on the location of pre-existing contacts. We aimed to recruit three participants from each location ( $n=21$ total). We were prepared to continue sampling until data saturation was reached and no new themes relevant to the research question were identified.

\section{Interview Guide}

The interview guide explored three overarching topics: (1) the impact of COVID-19 on the healthcare system and its capacity to respond, (2) information needs, access, sharing, and dissemination; and (3) direct impact of COVID-19 on participants. The interview guide was developed iteratively through a series of working group meetings that included research assistants (RBM, CD, ES, LK), a qualitative research 
expert (JPL), and a physician assistant $(\mathrm{CH})$. The interview guide was informed by news topics and clinical discussions, particularly grand rounds at a tertiary academic teaching hospital in Alberta, Canada through March 2020. We pilot tested the interview guide with three critical care physicians to ensure the questions were appropriate in content and flow [Appendix 2]. The interview guide was refined after each pilot interview and was designed to focus on the first wave of the pandemic. Changes to the interview guide included removing two questions which were found to be redundant and improving the transition between the ice breaker question and the first question. Interviews were scheduled for 30-minutes and were closed with soliciting suggestions to enhance the response including preparedness needs for the COVID-19 in their hospitals.

\section{Data Collection and Analysis}

One investigator (FR) emailed invitations to critical care physicians identified by two investigators (a senior consultant in critical care medicine, FR; and an expert in qualitative research, JPL). Participants provided written, informed consent prior to participating in the interview. Two investigators (CD, $C H$ ) conducted semi-structured interviews via telephone between April 7, 2020, and August 27, 2020. Investigators administered a short demographics questionnaire at the end of the interview [Appendix 2]. Audio recordings were transcribed verbatim by a professional transcription company (Rev.com) and were quality checked and deidentified (names and context) in duplicate by two co-authors (CD, CH). Participants had the option to complete the interview by returning a written response format of the semistructured questions. All participants were offered the opportunity to review their de-identified transcript as a form of member checking.

Two researchers (CD, AD) used NVivo12 (https://www.qsrinternational.com/) to manage the data and facilitate thematic analysis [17]. Two investigators (CD and AD) independently reviewed a single transcript to generate an initial list of codes based on developing patterns and key ideas. The same two investigators then collaboratively developed a coding framework based on the outcomes of the initial open coding. They further refined the coding framework on an additional three transcripts to amalgamate similar ideas and capture new ideas. A coding framework based on the outcomes of the open coding process was then collaboratively developed by the same two investigators, who continued to meet weekly after coding consecutive groups of three transcripts to discuss themes and coding discrepancies and refine the coding framework. With every adjustment to the framework, investigators re-coded previous interviews to maximize analytic integrity. The investigators conducted a secondary stratified analysis of textual data; one investigator (CD) analyzed by sex, marital status, and parental status, and the other investigator ( $A D)$ analyzed by age, country, and (private or public) health system. After independent analysis, investigators met to discuss findings and implications. Discrepancies in analysis were addressed through discussion in meetings between the coding investigators (CD, $A D)$ and a qualitative research expert (JPL). The manuscript was edited by all co-workers before final submission to the journal.

\section{Results}


Of the 32 critical care physicians that were emailed, 15 did not respond and 1 declined, where 16 (50\%) consented to be interviewed, representing seven countries (USA, Canada, Turkey, England, Scotland, Italy, Spain, and Pakistan) (Table 1). Six participants were interviewed during the first wave of the pandemic (Canada: $n=2$; Turkey: $n=1$; Spain, England and Scotland: each $n=1$ ), while ten participants were interviewed within the interim between the first and second waves of the pandemic (USA: $n=1$; Canada, Turkey, England, Scotland, Italy, Spain and Pakistan: each $n=1$ ). The interviews averaged 29.3 minutes (standard deviation, 9.7 minutes); one participant completed the interview by returning a written response format of the semi-structured questions. Nine (56\%) participants were female, and the median age of participants was 45.5 (interquartile range, $38.5,56.75$ ).

Physicians unanimously shared their perceptions on the importance of building from the COVID-19 pandemic to adequately prepare for future public health crises and events that may be associated with strain on healthcare systems. Within this data, researchers $(C D, A D)$ identified six recurring themes that included: 1) sourcing and implementation of trusted information; 2) health systems-level preparedness with accessible supports; 3 ) institutional adaptations including changes to patient care; 4) professional safety and occupational wellbeing; 5) triage and restricted visitation policies; and 6) managing personal familial responsibilities. Exemplary quotations are provided in Table 2.

\section{Sourcing and Implementation of Trusted Information}

Participants from all interviews provided their perspectives on the challenge of sourcing and implementing trusted information regarding COVID-19 pandemic. Most critical care physicians regarded the importance of reliable, evidence-based data: "I found the epidemiological data is the most helpful for understanding the most common patient, what they will look like." Some critical care physicians created intentional distance from informational platforms: "I deliberately made a disconnect because I would read all the time, the New York Times and the newspaper. I've stopped all that, deliberately because I don't want to see it," and "I don't rely too much on social media to gather information. I can't say that I spend too much time focusing on what's posted on social media or text threads or chats or that type of thing. I kind of take them with a grain of salt." However, despite creating boundaries, critical care physicians recognized the difficulty in navigating the challenging and changing pandemic: "It's not outward misinformation but trying to sort through a new disease for which there's been no previous precedent to work by." In addition, participants unanimously described the burden of having to constantly correct misinformation: "There's a lot of weird messages about masks. I think that misinformation is harmful. I feel the need to constantly correct any misinformation to my family, to my patient's family, which is exhausting." One critical care physician recounted their experience with misinformation on social media platforms:

There was so much junk in the media about doctors, usually male, in fact let's be honest, always male, who thinks that they have the answer to everything, and their latest wonder cure, many of which were then proven to be pretty worthless. 


\section{Health Systems-level Preparedness with Accessible Supports}

Participants shared their need to receive regular and clear information from leadership within their health systems regarding pandemic planning and policy changes. One critical care physician remarked on the clarity and efficiency of the preparedness within their health system: "Early on, I think there was a big anticipation of the surge, and so there was a lot of work that started in February from a zone, as well as from a provincial approach to be able to prepare for a unified front in terms of managing an anticipated surge" while another participant echoed: "When the [first] wave started there was a staged approachcoordinated, very clear communication. I think that the management of that went very smoothly actually in such a way that nobody was overwhelmed in terms of providing care." In contrast, one critical care physician described feeling distressed about the transmissibility of the virus that was exacerbated with lack of health system preparedness: "Just the emotional toll that it's taking on a personal level for people to be walking in these rooms where you know that there's a risk of having that disease transmitted on to yourself. So emotionally, I think people are probably a little bit more taxed, certainly than usual, we were not prepared." Participants unanimously commented on the lack of systems-level accessible supports for staff:

We didn't have staff testing at the time, so I have to rely just on symptomatology. I was really quite anxious about being a spreader. Could I spread this amongst my colleagues?

\section{Institutional Adaptations Including Changes to Patient Care}

All critical care physicians who participated shared logistical adaptations to patient care within their institutions: "Our ICU is now purely for coronavirus patients. We are now completely dedicated to coronavirus patients. Our operating suite recovery area has been turned into the non-coronavirus ICU, so patients go there if they require critical care services but are coronavirus negative." Some physicians described their experiences with being involved in pandemic response teams:

I recommended to the administration that we have to form what we call a COVID-19 team. That was basically infectious disease, pulmonologists, hospitalists, pharmacists, nurses, infection prevention. So, we would round on actually each patient quickly for one to two minutes and make up a treatment plan for that day, for that specific patient.

In the end, critical care physicians, tired and burnt out, described the impact of adaptations within their institution that at times involved drastic changes to patient care: "Care admissions are being treated as positive and isolated until we can prove they are negative. We never would've isolated patients until we proved they haven't got a disease."

\section{Professional Safety and Occupational Wellbeing}

Participants provided their perspectives on the challenge of adhering to policies that jeopardized their safety at work: "I think the problem of the mixed messages is in the beginning [of the pandemic]". From 
the hospital administration, they were getting scared they will have shortage of healthcare workers, or nurses, or physicians. So, the message was, even if you're sick, you can come to work." Critical care physicians described their experiences with having to adapt to a workforce that was reduced purposefully to maintain physician safety: "The decision our [hospital] leadership made was to remove the older folks. I think anyone 65 and older. Then we had two pregnant physicians at the time from service and I think that was a hard decision for them to make. It was a challenging decision across the board." One critical care physician described the impact of the pandemic on their training environment and career trajectory: "From a professional standpoint as a resident, it has taken us away from our training a little bit. Our training opportunities," while another shared a renewed sense of purpose in their clinical practice: "I mean, I think I would have had a really hard time if I was in a different specialty, or I wasn't able to directly participate because part of it is it gave me a sense of purpose and feeling like I was doing something for the greater good during a really bad time." All critical care physicians shared their perspectives on the unintended positive impact of the pandemic regarding shared lessons for future pandemic preparedness planning:

We've managed to recruit some of those [temporary] nursing staff to join our facility permanently. So, critical care at our site has come out of it [the pandemic] better equipped and much more cohesive, and more highly skilled with some additional workforce.

\section{Triage and Restricted Visitation Policies}

Critical care physicians shared that withholding and withdrawing life-sustaining treatment while mitigating suffering in the ICU during the COVID-19 pandemic was extremely complex. One participant in Spain reflected on the mental and ethical demands of triage: "We have to do a triage and that was the worst part and that was the really difficult part. I have hard time to deal with that because we sometimes we have to say no to patients that were 75 years old that were in a really good shape and no comorbid conditions. But I have another one of 68 that was in the same situation, and I had to decide to intubate the one of 68 and not intubated the one of 75 because we didn't have ventilators." Another participant made note of the impact of restricted visitation policies that prevented family members from visiting their loved ones at end of life: "To tell the family that the husband or the wife or the father was dying in the ICU and they cannot come to see them. They cannot hold their hand, they cannot be with them. And I don't know, that was really tough." The significance of patient's family members was conspicuous by their absence:

After I round in the morning, I have to come back to my office and I just call families on the phone or on Zoom. I find it exhausting and not nearly as personally satisfying as it would be if I was in the room talking to them or updating them in the ICU. It pulls me away from direct patient care because I' $m$ trying to update families and I think that's I would say pretty draining.

\section{Managing Familial Responsibilities}

Participants described their perspectives on the challenge of managing personal familial responsibilities as they battled with increased demands in the ICU. Difficult for all, this was especially burdensome on 
families with young children: "I was watching my wife suffering the quarantine alone with the two boys with a lot of energy and she hardly had any time to sleep. And she, we have a little one, two years old that he's not sleeping well and she was not sleeping well and I couldn't help her because I was in the hospital almost every day, almost 20 hours a day and I couldn't be at home." Most participants agreed about the guilt when absent from their home:

I'm a mom with a four-year-old and a one-year-old. My husband is a physician as well, so we initially had a lot of concerns about how we were both going to continue to work full time. That was, I think, a particularly unique challenge for me, different than many of my colleagues who do not have young children, and most of them are male.

Admirably, physicians took opportunities to be actively involved in the care of extended family: "My parents live on the other side of town. They're in their seventies and have the usual collection of comorbidities of people in their seventies, so they've not been out shopping, so I do the shopping for them and drop it off with them and so forth."

\section{Discussion}

We conducted a semi-structured interview study with critical care physicians across seven countries to explore how critical care physicians, their clinical practice, and the hospitals in which they work, were impacted by the initial stage of the COVID-19 pandemic across multiple countries to understand shared preparedness needs to inform future stakeholder driven pandemic preparedness planning for the COVID19 pandemic and future pandemics. Our findings indicated that changes and adaptations at the health system, institutional, and personal level, to control spread of the SARS-CoV2 virus, transformed the way critical care physicians cared for their patients and coped with the overwhelming emotional demands of the pandemic. These changes led to complex situations that have professional and personal consequences for physicians. The unintended consequences experienced by critical care physicians largely hinged on the notion that despite seeking evidence-based information and receiving updates from leadership, critical care physicians felt scared about the ICU, and felt guilt related to leaving their families at home for extended periods of time. Our data highlight the importance of creating a solid base after the COVID-19 pandemic from which we can adequately prepare for future public health crises and events that may be associated with strain on the healthcare system and increased burnout and compassion fatigue in critical care physicians.

Emerging research has demonstrated a significant emotional burden on frontline healthcare workers [4, $10]$, including critical care physicians $[14,18]$, during the COVID-19 pandemic. Potential resource shortages or circumstances out of physician's realm of (clinical) control were of particular concern to participants in our study [2]. This issue played out in real-time as some were forced to triage critical care resources for patients, due to a lack of solidified or actualized triage policies, and high patient volumes [19]. Participants in our study expressed the immense emotional difficulty in making these decisions, particularly in an environment where efficient decisions needed to be made. This moral distress is 
described in the literature where clinicians were fearful of having to ration resources [20,21]. Earlier studies have also demonstrated the need for ethical and legal parameters in triaging practices to support physicians in vulnerable triaging scenarios, particularly during crisis surge responses [15, 22, 23]. Given the prevalence of symptoms of depression, anxiety, and burnout among physicians [18] our data underpins the importance of attaining and maintaining good mental health and emotional wellbeing.

The preparedness of health systems to respond to the COVID-19 pandemic has been questioned by healthcare professionals all around the world $[14,15,24]$. In the current study participants spoke about the need to reorganize ICUs to accommodate COVID-19 patients, re-deploy high risk staff into administrative roles, or train additional staff in the support of critically ill patients. In 2007 the World Health Organization published a framework with six building blocks (i.e., service delivery, health workforce, information, medical products, vaccines and technologies, financing, and leadership and governance [stewardship]) toward strengthening health systems globally [25]. Researchers have since proposed methods of approach to health systems resilience during shock scenarios, wherein systems see a rapid increase in the volume of critically ill patients $[24,26,27]$. The COVID-19 pandemic has revitalized the need for global health systems to plan and prepare for possible scenarios of surge and emergency situations. Capturing the lived experiences of stakeholders across healthcare jurisdictions and infusing these findings into future pandemic preparedness planning is an attractive area for future work.

Our findings highlight that policy changes, while necessary from a public health perspective, had unintended but deleterious consequences on healthcare professionals working in the ICU during the COVID-19 pandemic. The COVID-19 pandemic resulted in limitations on family engagement in the ICU and participation in care that completely reengineered physician's methods to practice and had potential implications on their well-being [28-30]. Supporting patient's family members is foundational to the practice of critical care medicine [31] that is rarely easy [32] and has been more challenging in the COVID19 pandemic, especially at end-of-life [33]. This includes patients dying alone due restricted visitation policies in in the ICU-the detrimental implications of this reality for patients, families, and critical care physicians, cannot be understated [34, 35].

Our co-designed interview guide was informed by narratives reported in the COVID-19 pandemic [36-39] and tested in pilot interviews with critical care physicians. Interviews were conducted individually and at length, which allowed physicians' time and space to describe experiences to offer important insights on the psychological burden that afflicts their practice in the ICU. There are limitations to consider when interpreting the findings of our study. As cases of COVID-19 fluctuated globally, health systems, access to resources, and experiences of critical care physicians, may have differed, limiting the generalizability of our work. We purposively recruited critical care physicians from countries with varied case counts and health systems to create a more generalizable sample. However, as our participants were also not systematically sampled, and interviews were conducted in the English language, findings may not be broadly applicable. This study was conducted at the beginning of the pandemic prior to variants of concern, as well as the development and implementation of vaccinations. Our small sampling frame limited our ability to achieve data saturation in our analysis; it was difficult to recruit critical care 
physicians during the first wave of the pandemic. Additional interviews to collect data past code saturation in order to assess meaning saturation are required for transferability of our results [40].

\section{Conclusion}

The data from our semi-structured interview study with critical care physicians across seven countries indicate that changes and adaptations at the health system, institutional, and personal levels to control spread of the SARS-CoV2 virus transformed the way critical care physicians cared for their patients and coped with emotional demands of the COVID-19 pandemic. Contributing to the pandemic response, critical care physicians felt scared about the ICU as well as guilt about continuously leaving their families at home. Preparedness planning for future health crises and events that may be associated with strain on the healthcare system should include the experiences and perspectives of critical care physicians.

\section{Declarations}

\section{Ethics Approval and Consent to Participate}

The University of Calgary Conjoint Health Research Ethics Board (Ethics ID\#: REB20-0377) and Dalhousie University Research Ethics Board (Ethics ID\#: 2020-5106) approved this study.

\section{Consent for Publication}

Not applicable.

\section{Availability of Data and Materials}

The datasets used and/or analysed during the current study are available from the corresponding author on reasonable request.

\section{Competing Interests}

The authors are unaware of any competing interests.

\section{Funding}

This original research study is funded by the Canadian Institutes of Health Research Operating Grant: Socio-Cultural Implications of COVID-19: Educating, Engaging \& Empowering the Public (Application Number: 40241). The funders had no role in protocol design, data collection and analysis, decision to publish, or preparation of the manuscript; no financial relationships with any organizations that might have an interest in the submitted work in the previous three years; no other relationships or activities that could appear to have influenced the submitted work.

\section{Author's contributions}


Each author contributed to the study's conception, design and interpretation, and each has given substantial input to successive revisions of the manuscript. The final version has been seen and reviewed by all authors and they assume responsibility for the integrity of the data and the accuracy of the analysis.

\section{Acknowledgements}

We would like to thank Carmen Hiploylee, Research Assistant, for conducting qualitative interviews for this study.

\section{References}

1. Ng-Kamstra J, Stelfox HT, Fiest KM, Conly J, Parsons Leigh J. Perspectives on personal protective equipment in acute-care facilities during the COVID-19 pandemic. CMAJ. 2020;192(28):E805-E9.

2. Parsons Leigh J, Kemp LG, de Grood C, Brundin-Mather R, Stelfox HT, Ng-Kamstra JS, et al. A qualitative study of physician perceptions and experiences of caring for critically ill patients in the context of resource strain during the first wave of the COVID-19 pandemic. BMC Health Serv Res. 2021;21(1):374.

3. Maves RC, Downar J, Dichter JR, Hick JL, Devereaux A, Geiling JA, et al. Triage of Scarce Critical Care Resources in COVID-19 An Implementation Guide for Regional Allocation: An Expert Panel Report of the Task Force for Mass Critical Care and the American College of Chest Physicians. Chest. 2020;158(1):212-25.

4. Cag Y, Erdem H, Gormez A, Ankarali H, Hargreaves S, Ferreira-Coimbra J, et al. Anxiety among frontline health-care workers supporting patients with COVID-19: A global survey. Gen Hosp Psychiatry. 2021;68:90-6.

5. Haldane V, De Foo C, Abdalla SM, Jung AS, Tan M, Wu S, et al. Health systems resilience in managing the COVID-19 pandemic: lessons from 28 countries. Nat Med. 2021;27(6):964-80.

6. Kaye AD, Okeagu CN, Pham AD, Silva RA, Hurley JJ, Arron BL, et al. Economic impact of COVID-19 pandemic on healthcare facilities and systems: International perspectives. Best Practice \& Research Clinical Anaesthesiology; 2020.

7. Fiest KM, Krewulak KD, Hiploylee C, Bagshaw SM, Burns KEA, Cook DJ, et al. An environmental scan of visitation policies in Canadian intensive care units during the first wave of the COVID-19 pandemic. Can J Anaesth 2021.

8. Fiest KM, Parsons Leigh J, Krewulak KD, Plotnikoff KM, Kemp LG, Ng-Kamstra J, et al. Experiences and management of physician psychological symptoms during infectious disease outbreaks: a rapid review. BMC Psychiatry. 2021;21(1):91.

9. Hajjar LA, Costa I, Rizk SI, Biselli B, Gomes BR, Bittar CS, et al. Intensive care management of patients with COVID-19: a practical approach. Ann Intensive Care. 2021;11(1):36. 
10. Pappa S, Ntella V, Giannakas T, Giannakoulis VG, Papoutsi E, Katsaounou P. Prevalence of depression, anxiety, and insomnia among healthcare workers during the COVID-19 pandemic: A systematic review and meta-analysis. Brain Behav Immun. 2020;88:901-7.

11. Van Steenkiste E, Schoofs J, Gilis S, Messiaen P. Mental health impact of COVID-19 in frontline healthcare workers in a Belgian Tertiary care hospital: a prospective longitudinal study. Acta Clin Belg 2021:1-8.

12. Di Tella M, Romeo A, Benfante A, Castelli L. Mental health of healthcare workers during the COVID-19 pandemic in Italy. J Eval Clin Pract. 2020;26(6):1583-7.

13. Peng X, Meng X, Li L, Hu C, Liu W, Liu Z, et al. Depressive and Anxiety Symptoms of Healthcare Workers in Intensive Care Unit Under the COVID-19 Epidemic: An Online Cross-Sectional Study in China. Front Public Health. 2021;9:603273.

14. Wahlster S, Sharma M, Lewis A, Patel P, Hartog C, Jannotta G, et al. The coronavirus disease 2019 pandemic's effect on critical care resources and health-care providers: A global survey. Chest. 2021;159(2):610-33.

15. Phua J, Weng L, Ling L, Egi M, Lim C-M, Divatia JV, et al. Intensive care management of coronavirus disease 2019 (COVID-19): challenges and recommendations. The Lancet Respiratory Medicine. 2020;8(5):506-17.

16. Pendharkar SR, Minty E, Shukalek CB, Kerr B, MacMullan P, Lail P, et al. Description of a Multi-faceted COVID-19 Pandemic Physician Workforce Plan at a Multi-site Academic Health System. J Gen Intern Med. 2021;36(5):1310-8.

17. Braun VCV. Using thematic analysis in psychology. Qual Res Psychol. 2006;3(2):77-101.

18. Azoulay E, De Waele J, Ferrer R, Staudinger T, Borkowska M, Povoa P. Symptoms of burnout in intensive care unit specialists facing the COVID-19 outbreak. Annals of Intensive Care. 2020;10(1):110.

19. White DB, Lo B. A Framework for Rationing Ventilators and Critical Care Beds During the COVID-19 Pandemic. JAMA. 2020;323(18):1773-4.

20. Solomon MZ, Wynia MK, Gostin LO. Covid-19 Crisis Triage - Optimizing Health Outcomes and Disability Rights. N Engl J Med. 2020;383(5):e27.

21. Wynia MK. Crisis Triage-Attention to Values in Addition to Efficiency. JAMA Netw Open. 2020;3(12):e2029326.

22. Aziz S, Arabi Y, Alhazzani W, Evans LE, Citerio G, Fischkoff K. Managing ICU surge during the COVID19 crisis: rapid guidelines. Intensive Care Med. 2020;46(7):1303-25.

23. Arabi YM, Azoulay E, Al-Dorzi H, Phua J, Salluh J, Binnie A. How the COVID-19 pandemic will change the future of critical care. Intensive Care Med. 2021;47(3):282-91.

24. Lal A, Erondu NA, Heymann DL, Gitahi G, Yates R. Fragmented health systems in COVID-19: rectifying the misalignment between global health security and universal health coverage. The Lancet. 2021;397(10268):61-7. 
25. Organization WH. Everybody's business-strengthening health systems to improve health outcomes: WHO's framework for action. Geneva; 2007:44.

26. Blanchet K, Nam S, Ramalingam B, Pozo-Martin F. Governance and capacity to manage resilience of health systems: towards a new conceptual framework. international Journal of Health Policy Management. 2017;6(8):431-5.

27. Hanefeld J, Mayhew S, Legido-Quigley H, Martineau F, Karanikolos M, Blanchet K. Towards an understanding of resilience: responding to health systems shocks. Health Policy Plan. 2018;33(3):355-67.

28. Kent EE, Ornstein KA, Dionne-Odom JN. The Family Caregiving Crisis Meets an Actual Pandemic. J Pain Symptom Manage. 2020;60(1):e66-e9.

29. Cattelan J, Castellano S, Merdji H, Audusseau J, Claude B, Feuillassier L, et al. Psychological effects of remote-only communication among reference persons of ICU patients during COVID-19 pandemic. J Intensive Care. 2021;9(1):5.

30. Leggett A, Carmichael A, Leonard N, Robinson-Lane S, Li S, Oxford G, et al. Family Caregivers on the Frontline: Challenges of Providing Care to Post-ICU COVID-19 Patients. Innov Aging. 2020;4(Suppl 1):946-.

31. Davidson JE, Aslakson RA, Long AC, Puntillo KA, Kross EK, Hart J, et al. Guidelines for FamilyCentered Care in the Neonatal, Pediatric, and Adult ICU. Crit Care Med. 2017;45(1):103-28.

32. Brown SM, Azoulay E, Benoit D, Butler TP, Folcarelli P, Geller G, et al. The Practice of Respect in the ICU. Am J Respir Crit Care Med. 2018;197(11):1389-95.

33. Andrist E, Clarke RG, Harding M. Paved With Good Intentions: Hospital Visitation Restrictions in the Age of Coronavirus Disease 2019. Pediatr Crit Care Med. 2020;21(10):e924-e6.

34. Moss SJ, Krewulak KD, Stelfox HT, Ahmed SB, Anglin MC, Bagshaw SM, et al. Restricted visitation policies in acute care settings during the COVID-19 pandemic: a scoping review. Crit Care. 2021;25(1):347.

35. Moss SJ, Stelfox HT, Krewulak KD, Ahmed S, Anglin MC, Bagshaw SM, et al. Impact of restricted visitation policies in hospitals on patients, family members and healthcare providers during the COVID-19 pandemic: a scoping review protocol. BMJ Open. 2021;11(9):e048227.

36. Benatti SV. Love in the Time of Corona. Ann Intern Med. 2020;172(9):628.

37. Landry A, Ouchi K. Story of human connection. Emerg Med J. 2020;37(8):526.

38. Neville TH. COVID-19: A Time for Creative Compassion. J Palliat Med. 2020;23(7):990-1.

39. Life Lines Team c. Restricted family visiting in intensive care during COVID-19. Intensive Crit Care Nurs. 2020;60:102896.

40. Hennink MM, Kaiser BN, Marconi VC. Code Saturation Versus Meaning Saturation: How Many Interviews Are Enough? Qual Health Res. 2017;27(4):591-608.

\section{Tables}


Table 1

Demographic and clinical characteristics of the critical care physicians who agreed to participate in semi-structured interviews

\begin{tabular}{|c|c|}
\hline $\begin{array}{l}\text { Demographic and } \\
\text { Clinical Characteristics }\end{array}$ & $\begin{array}{l}\text { Critical Care Physicians } \\
(n=16)\end{array}$ \\
\hline \multicolumn{2}{|l|}{ Age category, years, n (\%) } \\
\hline $30-39$ & $4(25)$ \\
\hline $40-49$ & $6(37.5)$ \\
\hline $50-59$ & $3(19)$ \\
\hline $60-69$ & $2(12.5)$ \\
\hline $70-79$ & $1(6)$ \\
\hline Female, n (\%) & $9(56)$ \\
\hline \multicolumn{2}{|l|}{ Marital Status, n (\%) } \\
\hline Married & $15(94)$ \\
\hline Dependents, n (\%) & $14(88)$ \\
\hline Children & $13(92)$ \\
\hline \multicolumn{2}{|l|}{ Clinical Specialty, n (\%) } \\
\hline Critical Care & $8(50)$ \\
\hline Critical Care \& Anaesthesiology & $6(37.5)$ \\
\hline Infectious Disease & $1(6.25)$ \\
\hline Emergency Medicine & $1(6.25)$ \\
\hline \multicolumn{2}{|l|}{ Type of Institution, n (\%) } \\
\hline Academic & $14(88)$ \\
\hline Non-academic & $1(6)$ \\
\hline Community & $1(6)$ \\
\hline \multicolumn{2}{|l|}{ Country, n (\%) } \\
\hline Canada & $3(19)$ \\
\hline Spain & $2(12.5)$ \\
\hline Turkey & $2(12.5)$ \\
\hline Scotland & $2(12.5)$ \\
\hline
\end{tabular}




\begin{tabular}{|ll|}
\hline $\begin{array}{l}\text { Demographic and } \\
\text { Clinical Characteristics }\end{array}$ & $\begin{array}{l}\text { Critical Care Physicians } \\
(\boldsymbol{n}=16)\end{array}$ \\
\hline England & $2(12.5)$ \\
\hline USA & $2(12.5)$ \\
\hline Italy & $2(12.5)$ \\
\hline Pakistan & $1(6)$ \\
\hline
\end{tabular}


Table 2

Perspectives of critical care physicians on the lived experiences of working in the intensive care unit during the initial stage of the COVID-19 pandemic

\section{Themes Quotes}

\section{Sourcing and Implementation of Trusted Information}

"I deliberately made a disconnect because I would read all the time, the New York Times and the newspaper. I've stopped all that, deliberately because I don't want to see it."

"There was so much junk in the media about doctors, usually male, in fact let's be honest, always male, who thinks that they have the answer to everything, and their latest wonder cure, many of which were then proven to be pretty worthless."

"I found the epidemiological data is the most helpful for understanding the most common patient, what they will look like."

"I don't rely too much on social media to gather information. I can't say that I spend too much time focusing on what's posted on social media or text threads or chats or that type of thing. I kind of take them with a grain of salt."

"There's a lot of weird messages about masks. I think that misinformation is harmful. I feel the need to constantly correct any misinformation to my family, to my patient's family, which is exhausting."

"It's not outward misinformation but trying to sort through a new disease for which there's been no previous precedent to work by."

\section{Health}

Systems-level Preparedness with Accessible Supports

"Early on, I think there was a big anticipation of the surge, and so there was a lot of work that started in February from a zone, as well as from a provincial approach to be able to prepare for a unified front in terms of managing an anticipated surge."

"When the [first] wave started there was a staged approach-coordinated, very clear communication. I think that the management of that went very smoothly actually in such a way that nobody was overwhelmed in terms of providing care."

"Just the emotional toll that it's taking on a personal level for people to be walking in these rooms where you know that there's a risk of having that disease transmitted on to yourself. So emotionally, I think people are probably a little bit more taxed, certainly than usual, we were not prepared."

"We didn't have staff testing at the time, so I have to rely just on symptomatology. I was really quite anxious about being a spreader. Could I spread this amongst my colleagues?"

Institutional Adaptations Including Changes to Patient Care
"Care admissions are being treated as positive and isolated until we can prove they are negative. We never would've isolated patients until we proved they haven't got a disease."

"I recommended to the administration that we have to form what we call a COVID19 team. That was basically infectious disease, pulmonologists, hospitalists, pharmacists, nurses, infection prevention. So, we would round on actually each patient quickly for one to two minutes and make up a treatment plan for that day, for that specific patient."

"Our ICU is now purely for coronavirus patients. We are now completely dedicated to coronavirus patients. Our operating suite recovery area has been turned into the non-coronavirus ICU, so patients go there if they require critical care services but are coronavirus negative." 


\section{Themes}

Professional

Safety and Occupational Wellbeing

\section{Quotes}

"The decision our [hospital] leadership made was to remove the older folks. I think anyone 65 and older. Then we had two pregnant physicians at the time from service and I think that was a hard decision for them to make. It was a challenging decision across the board."

"I think the problem of the mixed messages is in the beginning. From the hospital administration, they were getting scared they will have shortage of healthcare workers, or nurses, or physicians. So, the message was, even if you're sick, you can come to work."

"From a professional standpoint as a resident, it has taken us away from our training a little bit. Our training opportunities."

"I mean, I think I would have had a really hard time if I was in a different specialty, or I wasn't able to directly participate because part of it is it gave me a sense of purpose and feeling like I was doing something for the greater good during a really bad time."

"We've managed to recruit some of those [temporary] nursing staff to join our facility permanently. So, critical care at our site has come out of it [the pandemic] better equipped and much more cohesive, and more highly skilled with some additional workforce."

Triage and Restricted Visitation Policies

"We have to do a triage and that was the worst part and that was the really difficult part. I have hard time to deal with that because we sometimes we have to say no to patients that were 75 years old that were in a really good shape and no comorbid conditions. But I have another one of 68 that was in the same situation, and I had to decide to intubate the one of 68 and not intubated the one of 75 because we didn't have ventilators."

"After I round in the morning, I have to come back to my office and I just call families on the phone or on Zoom. I find it exhausting and not nearly as personally satisfying as it would be if I was in the room talking to them or updating them in the ICU. It pulls me away from direct patient care because I'm trying to update families and I think that's I would say pretty draining."

"To tell the family that the husband or the wife or the father was dying in the ICU and they cannot come to see them. They cannot hold their hand, they cannot be with them. And I don't know, that was really tough."

Managing Familial Responsibilities
"My parents live on the other side of town. They're in their seventies and have the usual collection of comorbidities of people in their seventies, so they've not been out shopping, so I do the shopping for them and drop it off with them and so forth."

"I'm a mom with a four-year-old and a one-year-old. My husband is a physician as well, so we initially had a lot of concerns about how we were both going to continue to work full time. That was, I think, a particularly unique challenge for me, different than many of my colleagues who do not have young children, and most of them are male."

"I was watching my wife suffering the quarantine alone with the two boys with a lot of energy and she hardly had any time to sleep. And she, we have a little one, two years old that he's not sleeping well and she was not sleeping well and I couldn't help her because I was in the hospital almost every day, almost 20 hours a day and I couldn't be at home." 


\section{Supplementary Files}

This is a list of supplementary files associated with this preprint. Click to download.

- GlobalManuscriptAppendicesFINALBMCHSRversion.docx 